OPEN ACCESS

Edited by:

David H. Volle,

Institut National de la Santé et de la

Recherche Médicale, France

Reviewed by:

Adriana Maggi,

University of Milan, Italy

Anne Hélène Duittoz,

Université de Tours, France

*Correspondence:

Lavinia Casati,

Department of Medical Biotechnology and Translational Medicine, University

of Milan, Via Vanvitelli 32, 20129

Milan, Italy

lavinia.casati@unimi.it

Specialty section: This article was submitted to

Cellular Endocrinology,

a section of the journal

Frontiers in Cell and Developmental

Biology

Received: 30 January 2015 Accepted: 17 May 2015

Published: 18 June 2015

Citation:

Casati L, Sendra R, Sibilia $V$ and Celotti F (2015) Endocrine disrupters:

the new players able to affect the epigenome. Front. Cell Dev. Biol. 3:37.

doi: $10.3389 /$ fcell.2015.00037

\section{Endocrine disrupters: the new players able to affect the epigenome}

\author{
Lavinia Casati $^{1 *}$, Ramon Sendra ${ }^{2}$, Valeria Sibilia $^{1}$ and Fabio Celotti ${ }^{3}$ \\ ${ }^{1}$ Department of Medical Biotechnology and Translational Medicine, University of Milan, Milan, Italy, ${ }^{2}$ Departament de \\ Bioquímica i Biologia Molecular, Universitat de València, Valencia, Spain, ${ }^{3}$ Department of Pharmacological and Biomolecular \\ Sciences, University of Milan, Milan, Italy
}

Epigenetics represents the way by which the environment is able to program the genome; there are three main levels of epigenetic control on genome: DNA methylation, post-translational histone modification and microRNA expression. The term Epigenetics has been widened by $\mathrm{NIH}$ to include "both heritable changes in gene activity and expression but also stable, long-term alterations in the transcriptional potential of a cell that are not necessarily heritable." These changes might be produced mostly by the early life environment and might affect health influencing the susceptibility to develop diseases, from cancer to mental disorder, during the entire life span. The most studied environmental influences acting on epigenome are diet, infections, wasting, child care, smoking and environmental pollutants, in particular endocrine disrupters (EDs). These are environmental xenobiotics able to interfere with the normal development of the male and female reproductive systems of wildlife, of experimental animals and possibly of humans, disrupting the normal reproductive functions. Data from literature indicate that EDs can act at different levels of epigenetic control, in some cases transgenerationally, in particular when the exposure to these compounds occurs during the prenatal and earliest period of life. Some of the best characterized EDs will be considered in this review. Among the EDs, vinclozolin (VZ), and methoxychlor (MXC) promote epigenetic transgenerational effects. Polychlorinated biphenils (PCBs), the most widespread environmental EDs, affect histone post-translational modifications in a dimorphic way, possibly as the result of an alteration of gene expression of the enzymes involved in histone modification, as the demethylase Jarid1b, an enzyme also involved in regulating the interaction of androgens with their receptor.

Keywords: epigenetics, endocrine disruptors, polychlorinated biphenyls, androgen receptor (AR), histone demethylases, Jarid1b

\section{Epigenetics: What are the Mechanisms?}

Epigenetics represents the programming of the genome to express the appropriate set of genes in a space- time specific way during life (Zhang and Ho, 2011). Epigenetic patterns are created during cellular differentiation by a highly programmed and organized process (Casati, 2013). However, epigenetic mechanisms are dynamic and responsive to the environment, especially during the critical periods of embryonic development and early life (Casati, 2013). The epigenome is constituted by a set of chromatin players, such as DNA methylation, histone modifications and miRNA expression, that dynamically interact to define a correct transcriptomic profile 
(Fleisch et al., 2012). The effect of the epigenetic regulation can be an extensive change in cell gene expression, as occurs in several instances of DNA methylation, or the fine modulation of specific genes (Casati, 2013). In this review we have briefly considered the three main levels of the epigenome.

\section{DNA Methylation}

The genomic distribution of methylated DNA sequences is defined "methylome"; the "methylome" it is able to modify itself in function of the environment or the developmental stage. DNA methylation involves the covalent addition of a methyl group at position 5 of the pyrimidine ring of cytosine in CpGs dinucleotides, called CpG sites (Lister et al., 2009). DNA regions rich in CpG sites are known as CpG islands (Bird, 2002). In the human genome $60-80 \%$ of 28 million CpG dinucleotides are methylated (Lister et al., 2009). Unmethylated CpG islands are targets of transcription factors to start transcription. By contrast, the $\mathrm{CpG}$ sequences in inactive genes are usually methylated to suppress their expression (Belzil et al., 2013). For some transcription factors, for example, AP-2, c-myc, CREB/ATF, E2F, and NF-kB, DNA methylation abolishes access to promoter binding sites. However, this action mechanism seems to be true only for a subset of transcription factors (Kulis and Esteller, 2010). Current evidences support a second mechanism in which DNA methylation patterns correlate with chromatin structure and function. Active regions, characterized by an open chromatin structure, where genes are expressed, are associated with hypomethylated DNA sequences, whereas hypermethylated DNA is packaged in a more compact and inactive chromatin (Razin, 1998; Casati et al., 2010). A number of different proteins able to bind specifically to methyl-CG has been identified and shown to perform critical roles in the regulation of gene expression (Buck-Koehntop and Defossez, 2013). These proteins contains methyl-CpG binding domains (MBDs) which are stretches of about 75 amino acid residues long that are evolutionary conserved. Generally, DNA methylation seems to be a starting step for establishing the inactive chromatin state. It is followed by an MBD protein association that, in turn, recruits further repressive epigenetic modification enzymes, such as histone deacetylase (Kulis and Esteller, 2010) (see next section). The chromatin compacts and gene silencing is achieved. For example, a specific protein, $\mathrm{MeCP} 2$ (methylcytosine-binding protein 2) binds directly to methylated CG but not to unmethylated CG and its binding produces a tightly packed close chromatin structure and transcriptional repression. The importance of $\mathrm{MeCP} 2$ is shown by the finding that mutant $\mathrm{MeCP} 2$ forms, unable to recognize methyl-CG, produce the Rett syndrome, a severe developmental disorder leading to mental retardation (Adkins and Georgel, 2011).

DNA methyltransferases (DNMTs) are the enzymes involved in DNA methylation, of which at least three functional DNMTs have been identified in eukaryotic systems. DNMT1 is involved in maintenance of methylation status during replication (it can methylate only the CG sequence paired with methylated CG) (Reik et al., 2001); DNMT2 is related to embryonic stem cells and potential RNA methylation (Clouaire and Stancheva, 2008); and the DNMT3 family consisting of two members, DNMT3a and DNMT3b, which are involved in de novo DNA methylation at $\mathrm{CpG}$ sites occurring during early embryogenesis and are essential for the mammalian development (Singh and Li, 2012).

\section{Histone Modification}

The basic repeating unit of chromatin, the nucleosome, consists of $146 \mathrm{bp}$ of DNA wrapped around an octameric histone core formed by two copies each of histones H2A, H2B, H3, and H4 (Felsenfeld and Groudine, 2003). Histones beside possessing a definite structural function have a specific role in modulating the physical access of nuclear factors to DNA (Luger et al., 1997). Histones regulate the chromatin compaction degree: in this way they are able to regulate the transcriptional activity as well as transcriptional silencing (Kanherkar et al., 2014). How is it possible? It is now clear that post-translational modifications of charged aminoacids of histone tails that protrude from the nucleosome can alter chromatin conformation and create binding sites for transcription factors; in this manner they can play a direct regulatory role in gene expression (Felsenfeld and Groudine, 2003). There are a lot of histones post-translational modifications that involve mostly lysine, arginine, threonine and serine residues (Cheung and Lau, 2005; Casati et al., 2010). Among them, the modifications more extended are acetylation, methylation, phosphorylation, ubiquitination, sumoylation, and ADP ribosylation (Cedar and Bergman, 2009). It is therefore apparent that a very strong modulating activity can be produced by the many possible combinations of modifications that can occur on a variety of sites on histones (Cheung and Lau, 2005). Among all the post-translational modifications of histones, lysine methylation and acetylation of histones $\mathrm{H} 3$ and $\mathrm{H} 4$ (Fischle et al., 2003) are the best studied. Histone methylation is catalyzed by histone lysine methyltransferases (HKMTase), whereas histone acetyltransferase (HAT) and histone deacetylases (HDACs) regulate, respectively, the acetylation, and deacetylation of lysine residues (Szyf, 2009).

It is recognized that histone post-translational modifications can regulate DNA accessibility by two different, but not mutually exclusive, ways (Suganuma and Workman, 2011). In one model, post-translational modifications of histones directly modulate chromatin compaction states across changes on the physicochemical properties of the chromatin at the modification sites, thereby altering DNA-histone and histone-histone interactions within the nucleosomes or between nucleosomes. For example, acetylation of lysine residues neutralizes positive charges of histones and affects the electrostatic interactions between positively charged histones and negatively charged DNA. In the second way, histone post-translational modifications generate signaling platforms to recruit a variety of chromatin-binding proteins that recognize specific patterns of modifications on histones ("readers" or "effectors"), which subsequently lead to downstream cellular programs such as transcription modulation. Different protein domains have been identified that can recognize specific histone modifications, although they appear to be more flexible than the enzymes that create the modifications (Patel and Wang, 2013). For example, bromodomains recognize specifically 
acetyl-lysine residues on histones, whereas chromodomains bind methylated lysines, and tudor domains bind methylated arginines. Many evidences have revealed that histone posttranslational modifications can act as a heritable "code" (so-called "histone code") that can be passed during cell division to the progeny. Histone post-translational modifications could therefore permit the inheritance of phenotypic features independent of the DNA sequence. Given their involvement in fundamental cellular processes, dysfunction of histone posttranslational modifications is found in diverse human diseases, particularly in cancer (Chi et al., 2010).

\section{RNA Interfering}

The third epigenetic mechanism is the post-trascriptional RNA induced silencing mediated by small, non-coding RNAs which down-regulate or suppress expression of specific genes. The silencing process is operated by microRNAs (miRNAs) and by short interfering RNAs (siRNAs); they are both 20-30 nucleotide-long double-stranded RNA molecules, encoded by their own set of cellular genes (miRNAs) or introduced into the cell from outside sources (siRNAs), e.g., virus (Carthew and Sontheimer, 2009). Although microRNAs only represent $1 \%$ of the genome, they have been estimated to mark $30 \%$ of genes (Lewis et al., 2005). These RNAs can act as switches and modulators, exerting extensive influence within the cell, fine-tuning the gene expression in specific cell-types during development as well as in pathological conditions (Baer et al., 2013). MicroRNAs have also been shown to play a role in cancer inhibition, apoptosis, cellular proliferation and cell movement suggesting that they can be used to supply an epigenetic cure to cancer (Kala et al., 2013).

\section{Epigenetics and Environment: Focus on the Environmental Factors able to Shape the Epigenome}

The epigenetic changes might be produced by the environmental condition during the prenatal and early life and might influence the susceptibility to develop several diseases, from cancer to mental disorder during the entire life span (Foley et al., 2009). The epigenome integrates the informations present in the genome with the environmental cues, establishing the trascriptomic profile typical for each cell type to define its functional identity. The epigenome characterizes the capability of an organism to adapt and evolve regulating the characteristics or phenotypes developed in response to environmental cues (Rivera and Ren, 2013). The most studied environmental cues able to affect the epigenome are diet, child care, smoking, infections, wasting and environmental pollutants, especially endocrine disrupters (EDs) (Casati et al., 2010).

\section{Prenatal Life}

Gestation represents a very sensitive period in epigenetic remodeling and a lot of scientific evidences underlined the importance of parental influences on the offspring epigenome. Maternal health can determinate childhood development and adult health condition, defining the susceptibility to develop a disease during the adult life (Kanherkar et al., 2014). In particular, fetal programming expresses the way by which the uterine environment affects the fetal molecular development through epigenetic mechanism (Schulz, 2010).

One example is the influence of maternal diet and war stress on offspring epigenome exemplified by the famous "Dutch famine birth cohort." It consists of more than 2000 singletons who were born between November 1943 and February 1947 in Amsterdam and systematically followed up since 1996 (El Hajj et al., 2014). Under the Nazi embargo of the Western Netherlands in 1944, pregnant women were under a severe nutritional restriction (El Hajj et al., 2014). Individuals who had been exposed to malnutrition and stress, during their early embryonic development, exhibited an increased risk for metabolic, cardiovascular and other complex diseases, schizophrenia, and accelerated cognitive aging (Schulz, 2010). More than 60 years after early gestational exposure, the Dutch famine individuals showed a subtle hypomethylation of the imprinted IGF2-H19 locus, compared with their unexposed siblings (Heijmans et al., 2008). A follow-up study on the same cohort reported alterations dependent on sex and exposure length, in the DNA methylation status of several imprinted and non-imprinted genes in blood cells (Tobi et al., 2009). Likewise in experimental animal models as much diet as stress conditions of the mother affect the epigenetic signature during the fetus development (Kanherkar et al., 2014). In this sense, Barua and colleagues have shown that a maternal folic acid supplementation induces in the offspring a different global DNA methylation profile from that in the offspring of mice which received a low folic acid dosage (Barua et al., 2014). Specifically, a distinct DNA methylation status was observed on genes associated with autism spectrum disorder (ASD) pathogenesis (Barua et al., 2014). Furthermore, paternal influences can also affect the epigenome of the offspring. It has been shown, in animal models, that the as much the alcohol consumption as exposure to toxic chemicals, such as chromium chloride and vinclozolin, by the paternal progenitors affect DNA methylation in germinal cells (Stouder and Paoloni-Giacobino, 2010). Similarly, subjecting male mice to folate deficiency resulted in an alteration of sperm function related to the differential DNA methylation in comparison to control mice (Leonard et al., 2004; Lambrot et al., 2013). As well, the male offspring of such mice deficient in folate also showed an altered transcriptomic profile in comparison to the offspring from control mice with a normal folate supplement (Lambrot et al., 2013).

\section{Perinatal Influences}

A wide variety of environmental effects play an important role after birth. Particularly important appears to be the interplay between epigenetics and social influences. Environmental experiences, specially early life adversities, as low maternal care in rats, produce increased promoter DNA methylation of the glucocorticoid receptor (GR) in the hippocampus 
causing differences in its expression (Weaver et al., 2004). Such gene expression alteration results in blunted negative feedback inhibition by glucocorticoids and a heightened stress response that continues into adulthood (Weaver et al., 2004). Epidemiological data suggest that the effects of early life adversity involve many genes and are not limited to rodents (McGowan et al., 2008). In post-mortem hippocampal samples from humans who were abused in childhood, the promoters of ribosomal RNA (rRNA) and GR genes were found hypermethylated in correlation with their low expression (McGowan et al., 2008, 2009).

\section{Adult Life}

During the adult life, environment is able to shape the epigenome and there are many factors that can affect it such as diet, caloric restriction, alcohol consumption, and environmental pollutants. Several studies have shown how diet affects DNA methylation patterns (Jennings and Willis, 2015). Since foods are able to alter epigenetic expression, nutrients extracted from the diet could be utilized to influence disease susceptibility.

\section{Nutraceuticals}

Micronutrients such as folate, retinoic acid, selenium compounds, polyphenols found in green tea, apples, coffee, black raspberries, and also other dietary sources containing genistein, soy isoflavones, curcumin, and resveratrol are able to influence epigenetic mechanisms and could be considered chemopreventive agents (Gerhauser, 2013).

One of the most studied epigenome modifiers is the phytoestrogen genistein, a bioactive isoflavone found in soy products, which is able to modulate the activity of DNA methyltransferases (Zhang and Chen, 2011). Genistein seems to affect tumorigenesis through epigenetic regulations, both histone methylation and DNA methylation (Zhang and Chen, 2011). Particularly, genistein appears to act, by modulating chromatin configuration and DNA methylation, activating tumor suppressor genes and affecting cancer cell survival (Zhang and Chen, 2011).

The polyphenol epigallocatechin-3-gallate (EGCG), present in green tea, is another example of micronutrient with antioxidant and chemopreventive properties (Fang et al., 2007), for which has been shown to slow down the carcinogenesis (Fang et al., 2007). The molecular mechanism of how EGCG works, inhibiting cancer cell growth, seems to be similar to that of other nutraceuticals such as soy genistein, and it involves DNA methylation regulation on key genes promoting positive epigenetic effects. Other bioactive compounds also considered as nutraceuticals are the sulfopropanes, present in and green tea and cruciferous vegetables, which have been associated with lower risk of cancer and other age-related diseases (Tollefsbol, 2014). It has been demonstrated that these compounds, in fact, have the capability to revert an aberrant epigenetic pattern (Tollefsbol, 2014).

\section{Environmental Chemicals}

Environmental pollutants, such as pesticides, are able to induce changes of DNA methylation in adults and also influence the susceptibility to different pathologies in offspring exposed during prenatal and early life (Kanherkar et al., 2014).

Widespread environmental contaminants belonging to heavy metal category, such as nickel, arsenic and cadmium disrupt normal histone acetylation and DNA methylation patterns, and have been related to different pathologies including tumorigenesis, neurological disorders, and autoimmune diseases (Leonard et al., 2004). A mode of action for these compounds has been hypothesized and may involve the fact that metals stimulate free radicals production inducing epigenetic alteration (Babar et al., 2008). For example, since S-adenosyl methionine (SAM), the universal methyl donor for methyltransferases (including DNMTs) is involved in arsenic detoxification (by methylation), arsenic exposure decreases the DNMTs activity but also, as has been shown, down-regulates DNMT gene expression (Reichard et al., 2007). Moreover, arsenic exposure induces hypermethylation of tumor suppressor genes (Jensen et al., 2008), disruption of histone acetylation (Hou et al., 2012), and upregulation of miRNAs expression (Marsit et al., 2006). Likewise, nickel is able to stimulate DNA methylation of tumor suppressor genes (Lee et al., 1995), to condense chromatin and to affect histone acetylation, which is accompanied of gene silencing; these effects finally lead to cell transformation (Zhang and Zhu, 2012). It is worth noting, as a possible mechanism of action, that a study has shown that $\mathrm{Ni}$ binds to N-terminal tails of histone $\mathrm{H} 4$ and, promoting a secondary structure with organized side-chain orientation, decreases the ability of histone acetyltransferase to recognize and bind to the histone tail and thus affects the ability of the enzyme to further modify the lysine residues (Zoroddu et al., 2010).

\section{EDs: the New Players able to Affect the Epigenome}

There are environmental xenobiotics that can interfere with the normal development of male and female reproductive systems of wildlife and experimental animals, and very probably of humans, disrupting endocrine axis in adulthood. These compounds are defined as endocrine disrupters (EDs). The exposure to EDs plays a key role on the epigenome shaping of many aspects of the endocrine function (Casati, 2013). The evidences present in the literature indicate that EDs can affect the different levels of epigenetic control and in some cases can act transgenerationally, if the exposure to EDs occurs during the prenatal and early life. There are several EDs which can act on epigenome in multiple ways (Casati et al., 2012). Several enzymes involved in epigenetic key processes of regulation of the endocrine system, such as histone-modifying enzymes, are altered either directly in their catalytic power or in their expression levels by EDs (Casati, 2013). It is also known that nuclear steroid receptors interact with histone-modifying enzymes to regulate gene transcription and chromatin compaction (Leader et al., 2006). 
Interestingly, histone demethylases (the enzymes responsible of the removal of the methyl groups from histones) take part in protein complexes together with steroid receptors, in particular with the androgen receptor (AR), facilitating the transcription of their target genes (Gao and Alumkal, 2010). Among the known EDs vinclozolin (VZ), a fungicide with antiandrogenic activity, and methoxychlor (MXC), an organochlorine pesticide actively metabolized into a derivative with a potent estrogenic activity, promote epigenetic transgenerational effects. $\mathrm{VZ}$ administerd during gestation promotes a male germline epigenome reprogramming, which probably induces transgenerational adult-onset diseases, disrupting DNA methylation patterns in sperm of the $\mathrm{F} 1$ generation of animals lasting at least up to F3 generation. MXC exposure in female rats, is also able to produce differentially DNA methylated regions (DMR), termed epimutations, in sperm epigenome, however the increased disease incidence in F4 generation reverse (female) outcross offspring indicated that the transgenerational disease transmission is primarily through the maternal germline (Manikkam et al., 2014).

Therefore, environmentally induced epigenetic transgenerational transmission can involve either the male and/or female germ cells and in mammals occurs at the later stages of primordial germ cell migration and colonization of the fetal gonad and during the initial stages of gonadal sex determination (Skinner et al., 2013). It is possible that DMR occur after the erasure of DNA methylation prior to gonadal sex determination and then subsequent re-methylation in a sex-specific manner (Skinner et al., 2010). Interestingly, the transgenerational effects disappear gradually from F1 to F3. Transmission of the altered germline epigenome (DNA methylation) to subsequent generations in an imprinted-like manner produces an altered epigenome and transcriptome in all cell types and tissues that develop from the maternal or paternal germlines having an altered epigenome (Guerrero-Bosagna et al., 2012; Manikkam et al., 2014).

For example vinclozolin (VIN) exposure, tested at doses that are environmentally-relevant, produces testicular, ovarian diseases, reproductive anomalies but also affects sociosexual, anxiety, cognition, appetitive, and locomotor behaviors in several animal species including birds, rodents, fish, amphibians (Crews et al., 2012; Guerrero-Bosagna et al., 2013; Leon-Olea et al., 2014). The behavioral alterations may be partially attributable to VINinduced alterations in gene expression (such as Gn-RH1, Esr1, Esr2, and Ar) in several hypothalamic nuclei, hippocampus, and striatum.

As mentioned above, one of the most studied EDs acting as an epigenome modifier is the phytoestrogen genistein, which is known to affect activity of DNA methyltransferases (Zhang and Chen, 2011). Moreover, modifications of the DNA methylation pattern in animals exposed to the synthetic estrogen diethylstilbestrol (DES) have been found (Casati et al., 2012). Likewise exposure to the pollutant bisphenol A (BPA), a plasticizer, also disrupt the DNA methylation pattern in agouti mice (Casati et al., 2012). Such BPA effect is reversible through food supplementation with methyl donor groups present in folate and genistein (Dolinoy et al., 2006).
Among the EDs there are the polychlorinated biphenyls compounds (PCBs) that are widely present in the environment (Casati et al., 2012). They were extensively used as dielectric and coolant fluids, i.e., in capacitors, transformers and electric motors (Colciago et al., 2009). Due to their toxicity and persistency in the environment, PCBs production was forbidden by USA in 1979 and by the Stockholm Convention on Persistent Organic Pollutants in 2001 (Colciago et al., 2006). PCBs are a group of 209 congeners with a broad spectrum of biological and toxic effects (Bonfanti et al., 2014). PCBs are classified as dioxin like (DL-PCBs), and non-dioxin compounds (NDL-PCBs) in function of their biochemical property (Casati et al., 2012). Many effects of DL-PCBs are mediated by the binding to the arylhydrocarbon receptor (AhR), a transcription factor present in many cell types of different animal species, including humans (Casati et al., 2012). The differential effects of DL- and NDLPCBs present in the environment are indistinguishable in vivo, since animals and humans are exposed simultaneously to both classes and the final effect is cumulative (Casati et al., 2012). Although PCBs production was terminated in '70, they are still present in the environment and chronic low-level exposure to $\mathrm{PCBs}$ represents a significant public health issue (Casati et al., 2012). A lot of studies show that PCBs exposure causes endocrine, metabolic and behavioral effects in animals and humans (Colciago et al., 2009). Moreover, it has been shown that PCBs might alter directly the transcriptomic profile, particularly during development (Tabb and Blumberg, 2006). In addition, recent data show that $\mathrm{PCBs}$ are also able to disrupt epigenetic mechanisms (Casati, 2013). It appears that exposure of pregnant rats to a $\mathrm{PCB}$ mixture, throughout the period of gestation, reduces expression and activity of DNMTs in liver of the offspring (Desaulniers et al., 2009). Furthermore, our previously published results showed that the exposure to a reconstituted mixture of PCBs (PCB 126, 138, 153, and 180) during gestation induces the expression of Jarid1b (a histone H3K4me3 demethylase) and SIRT1 (a histone H4K16ac deacetylase), and consequently a reduction of $\mathrm{H} 3 \mathrm{~K} 4 \mathrm{me} 3$ and $\mathrm{H} 4 \mathrm{~K} 16 \mathrm{ac}$ levels, in the liver of the offspring (Casati et al., 2012). The same exposed animals were characterized by a decrease of $\mathrm{AR}$ gene and protein expression (Casati et al., 2012).

Since steroid receptors can act as cofactors of histone modifying enzymes, we analyzed in some details, the PCBsAR-Jarid1b interaction (Casati et al., 2013). Therefore, we investigated: (1) how PCBs can affect the AR/Jarid1b interaction in the transcription of AR target genes; and (2) how PCBs affect $\mathrm{AR} /$ Jarid $1 \mathrm{~b}$ interaction in modulating the AR negative autofeedback. Above all we considered: (a) the potentiating effect of Jarid1b on AR transactivation induced by PCBs (Casati et al., 2012); (b) the role of PCBs and Jarid1b in the transcriptional activity of different $A R$ poly $Q$ variants (AR isoforms with different transactivation capability); and (c) the molecular mechanism exerted by Jaridlb in the AR transactivation, and the interaction with the AR promoter (Casati et al., 2013). PCBs treatment, in a dose-dependent manner, promotes AR transcriptional activity although its effect is lower than that produced by the natural ligand dihydrotestosterone (DHT). DHT represents the active $5 \alpha$-reduced testosterone metabolite, since 
possesses a higher affinity for AR than testosterone (Casati et al., 2013). Ligand binding studies have shown a specific and direct binding of several PCBs congeners to the ligand-binding domain of the AR protein (Portigal et al., 2002). Furthermore, Jarid $1 \mathrm{~b}$ is able to modulate the effects of AR-ligand interaction (Casati et al., 2013). The interplay between Jarid1b and AR on AR transactivation has been described in prostate cancer cells where Jarid1b is found up-regulated (Xiang et al., 2007). Likewise our previous in vivo studies have revealed that exposure to a mixture of PCBs stimulates the Jarid1b expression in the rat liver, and in its turn Jaridlb potentiates AR transcriptional activity (Casati et al., 2012). Moreover, in our more recent studies we have found that the overexpression of Jarid1b cotransfected with AR increases transcriptional activity induced by the treatment with DHT or PCBs in three different cell types: HEK293, and two neuronal cell lines, NSC34 and GN11 (Casati et al., 2013), indicating that the effect of the presence of Jarid $1 \mathrm{~b}$ on AR transactivation is not dependent on ligand or cell-phenotype. In spite of the described studies, the mode of action by which Jarid $1 \mathrm{~b}$ is able to modulate positively the AR transcriptional activity remains still uncertain. It is known that preservation of the enzymatic activity of Jarid $1 b$ is necessary for the increase of the AR transactivation, since the deletion of the JmjC domain, the demethylase catalytic center, eliminates the stimulation (Xiang et al., 2007).

PCBs-AR interaction is also modulated by differences in the structure of the AR gene found among individuals (Casati et al., 2013). It is known that AR transactivation is in part dependent on the length of a polyglutamine tract (polyQ, coded by a CAG repeat) located in the AR trans-activating region. The $\mathrm{CAG}$ repeat number in AR gene vary between 8 and 30 units in human populations, and thus the coded polyQ also is polymorphic in length (Ackerman et al., 2012). An inverse relationship has been found between the AR transcriptional activity and the length of poly Q repeat (Buchanan et al., 2004). Two recent reports by Bjork and coworkers show that PCBs have a CAG/PolyQ length dependent effect on AR in vitro (Bjork and Giwercman, 2013), and in some human prostatic cells (Bjork et al., 2011). In particular, PCB 153, present also in the reconstituted mixture used in our studies, has more pronounced effect on the in vitro AR transcriptional activity of short poly $\mathrm{Q}$ isoforms (Bjork et al., 2011). It is possible to hypothesize that Jarid1b-AR interaction affects the differential transcriptional activity of the $\mathrm{AR}$ isoforms, induced by PCBs, dependent on the interaction strength, which is lower for the long isoforms possessing a longer polyQ expansion (Casati, 2013). Moreover, Suzuki and coworkers have shown that the aberrant polyQ expansion potentiates the association between Rbp (Retinoblastoma Protein) and AR, and this association seems to attenuate the enrolment of HDAC1 (a histone deacetylase, class 1 ), which acts a potent transcription cofactor (Suzuki et al., 2009). It is possible that a similar mechanism could lead to the minor interaction shown in our studies for the longest isoform ARQ46, and consequently a low AR transactivation induced by PCB (Casati et al., 2013). On the contrary, the higher activation by the shorter AR isoforms seems to be mediated by a stronger interaction of this receptor with Jarid1b (Casati et al., 2013).

\section{A Hypothesis: How the EDs Could Affect the Epigenome? A Link through Steroid Receptor and Histone Demethylases}

The intersection between nuclear receptor activity and the epigenetic apparatus has several implications in the mode of action of endocrine disrupters. Since there are many classes of $\mathrm{ED}$, it is possible to hypothesize several ways by which ED could affect epigenetic mechanisms (see Figure 1).

Foremost it is possible that the alteration of DNA methylation activity induced by a single compound could be a common contributing factor to the dysregulation of several genes able to produce diverse phenotypic profiles (Anderson et al., 2012). The exposure to DES, an estrogenic ED, is a good example of this mechanism, since in animal studies the exposure to DES has been associated with a range of cancers, malformations of the genital track, and obesity (Newbold, 2011), probably related to alteration in DNA methylation pattern (Sato et al., 2009). Moreover, there are implications for direct or indirect longterm effects deriving from epimutations or aberrant epigenetic function (Anderson et al., 2012). For example an exposure in utero to some phthalates has been involved in the disruption of several steroidogenic pathway genes, contributing indirectly to malformations in offspring caused by the alteration of hormonal activity at critical developmental time points (Wilson et al., 2008). It is also possible that exposure to environmental pollutants affect histone methylation balance that indirectly produce longterm effects (Anderson et al., 2012). A permanent aberrant methylation might compromise the modulation of affected genes to future environmental cues, and thus increase susceptibility to develop disease during the entire life (Anderson et al., 2012). The rationale behind our hypothesis, linking epigenetic effects, EDs and steroid receptors, is that both short-term, indirect long-term and direct long-term effects might share a common etiology that involves, in part, nuclear-receptor mediated changes in histone methylation status (Anderson et al., 2012).

A paper from Wong and colleagues indicate that the perinatal exposure to EDs, and in particular xenoestrogens, increases cancer affecting the levels of DNA and histone methylation (Wong and Walker, 2013). Zhang and colleagues have analyzed the way by which xenoestrogens affect the epigenetic mechanism to reprogram the epigenome during the development (Zhang and Chen, 2011). These studies indicated that xenoestrogens induce non-genomic ER signaling activating PI3K/AKT pathways, resulting in AKT phosphorylation and inactivation of the histone methyltransferase $\mathrm{EZH} 2$, thus providing a direct relation to epigenome disruption (Zhang and Chen, 2011). Finally, it cannot be ruled out that non-genomic signaling could target also other epigenetic machinery components, suggesting this is a possible mechanism by which EDs could disrupt the epigenome (Wong and Walker, 2013).

On the other hand, according to our results, PCBs mediate a direct interaction between the histone demethylase Jarid1b and AR (Casati et al., 2013). The AR/Jarid1b binding on DNA of target gene seems to be allowed by the presence of Androgen Responsive Element (ARE) and by the presence of binding sites 


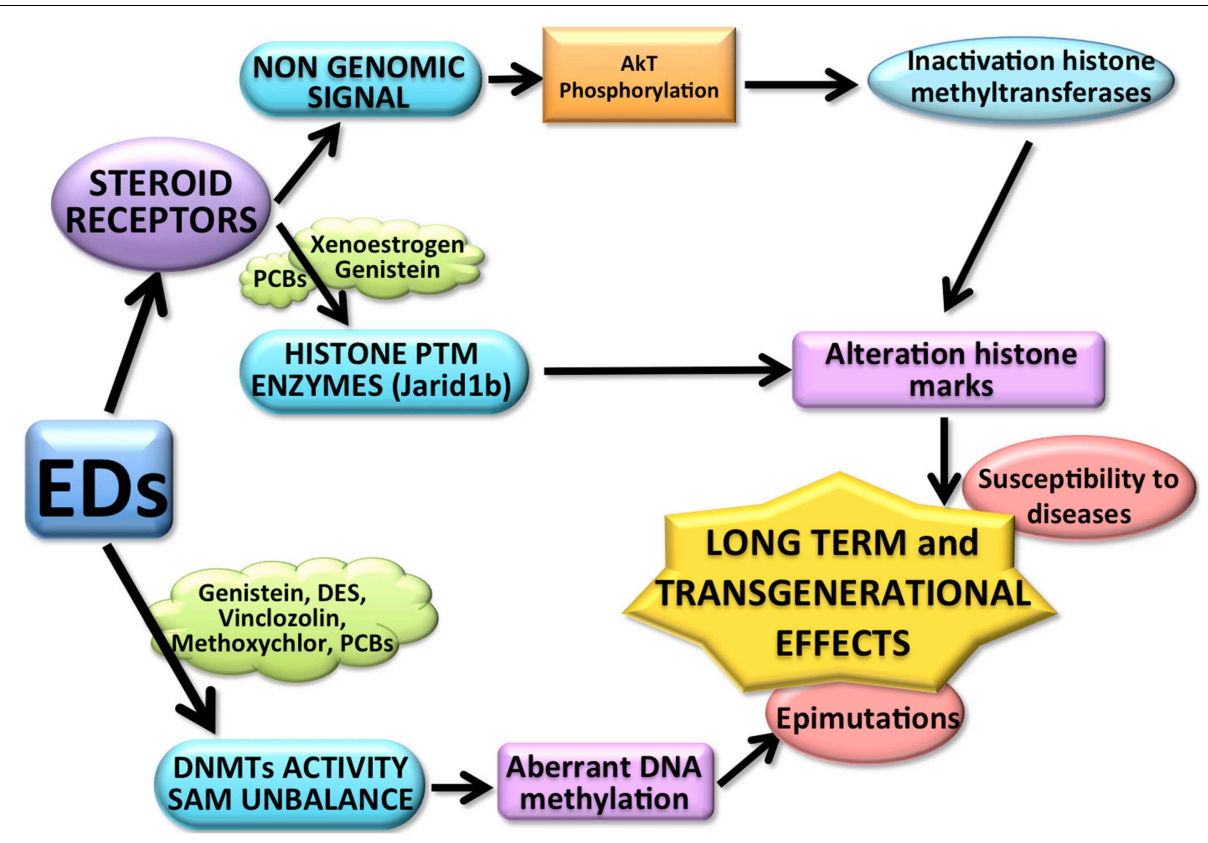

FIGURE 1 | The above diagram shows some of the possible ways by which EDs could affect epigenetic mechanisms. EDs, for example PCBs, (through the interaction with the steroid receptors) might affect directly histone post-translational modification (PTM) enzymes but also inactivate the histone modification enzymes through a non-genomic signal pathways (for example Genistein). Both mechanisms can affect the histone modification balance, causing long term effects. EDs (such as DES, Vinclozolin, Methoxyclor, PCB, and Genistein) can also induce an aberrant DNA methylation pattern affecting not only the activity of DNMTs, but also changing the availability of S-adenosyl-methionine (SAM). The resulting aberrant methylome causes epimutations, possibly transmitted transgenerationally and might increase the susceptibility to develop diseases. for the histone demethylases enzyme, Jarid1b (PLU1) and AhR (XRE), (Casati et al., 2013). As a matter of fact, in our previous studies, where the AR promoter DNA sequence was analyzed, we observed the presence of binding sites for Jarid1b (PLU1), some AREs, and XREs (Casati et al., 2013) [which at the same time suggests for a potential direct effect of the Jarid1b in modulating also the AR negative auto feedback (Vismara et al., 2009)]. In order to study the AR/Jarid1b interaction, we performed a series of gene reporter assays, where HEK293 cells were cotransfected with plasmids encoding for the luciferase gene, under the control of AR promoters with different lengths (long, intermediate and short), and the Jarid1b gene (Casati et al., 2013). Results showed that the presence of Jarid1b and, at least, two PLU1 binding sites are necessary for PCB-induced transactivation (Casati et al., 2013). We have hypothesized the involvement of Jarid1b as essential component for the interactions in the AhR-AR complex, occurring after exposure to PCBs, in particular in presence of DL congeners, since the responsive element XRE, ARE and PLU1 are concomitantly present on promoters of AR target genes.

\section{References}

Ackerman, C. M., Lowe, L. P., Lee, H., Hayes, M. G., Dyer, A. R., Metzger, B. E., et al. (2012). Ethnic variation in allele distribution of the androgen receptor (AR) (CAG)n repeat. J. Androl. 33, 210-215. doi: 10.2164/jandrol.111. 013391
Nevertheless, the relation between AR and AhR is complex and not fully understood (Kollara and Brown, 2010) and, to our knowledge, there is no data about a direct interaction between AhR and Jarid1b (Casati, 2013). It is possible to conclude that the AR modulation induced by PCBs involves AR-Jarid1b interactions. Further studies are needed to corroborate this hypothesis involving a delicate interplay between environment, epigenome and endocrine system (Casati, 2013).

\section{Conclusions}

Perturbation of nuclear receptors and epigenetic players may be a common mechanism in the epigenome modification caused by EDs. As matter of fact, even if the target genes affected by the endocrine disrupters may differ, the underlying mechanism, such as the perturbation of the delicate interplay between the actions of different epigenetic participants seems to be a common action mode (Anderson et al., 2012). Further studies will be necessary to delineate in a more precisely way the mechanism by which EDs are able to modify the epigenome.
Adkins, N. L. and Georgel, P. T. (2011). MeCP2: structure and function. Biochem. Cell Biol. 89, 1-11. doi: 10.1139/O10-112

Anderson, A. M., Carter, K. W., Anderson, D., and Wise, M. J. (2012). Coexpression of nuclear receptors and histone methylation modifying genes in the testis: implications for endocrine disruptor modes of action. PLOS ONE 7:e34158. doi: 10.1371/journal.pone.0034158 
Babar, I. A., Slack, F. J., and Weidhaas, J. B. (2008). miRNA modulation of the cellular stress response. Future Oncol. 4, 289-298. doi: 10.2217/14796694.4.2.289

Baer, C., Claus, R., and Plass, C. (2013). Genome-wide epigenetic regulation of miRNAs in cancer. Cancer Res. 73, 473-477. doi: 10.1158/0008-5472.CAN-123731

Barua, S., Kuizon, S., Chadman, K. K., Flory, M. J., Brown, W. T., and Junaid, M. A. (2014). Single-base resolution of mouse offspring brain methylome reveals epigenome modifications caused by gestational folic acid. Epigenetics Chromatin 7:3. doi: 10.1186/1756-8935-7-3

Belzil, V. V., Gendron, T. F., and Petrucelli, L. (2013). RNA-mediated toxicity in neurodegenerative disease. Mol. Cell. Neurosci. 56, 406-419. doi: 10.1016/j.mcn.2012.12.006

Bird, A. (2002). DNA methylation patterns and epigenetic memory. Genes Dev. 16, 6-21. doi: 10.1101/gad.947102

Bjork, C., and Giwercman, Y. L. (2013). Androgen receptor CAG repeat length modifies the effect of 2,3,7,8-tetrachlorodibenzo-p-dioxin on receptor activity in human prostate cells. Reprod. Toxicol. 35, 144-149. doi: 10.1016/j.reprotox.2012.10.010

Bjork, C., Nenonen, H., Giwercman, A., Bergman, A., Rylander, L., and Giwercman, Y. L. (2011). Persistent organic pollutants have dose and CAG repeat length dependent effects on androgen receptor activity in vitro. Reprod. Toxicol. 32, 293-297. doi: 10.1016/j.reprotox.2011.06.075

Bonfanti, P., Comelli, F., Assi, L., Casati, L., Colciago, A., Villa, S., et al. (2014). Responsiveness of hepatic and cerebral cytochrome P450 in rat offspring prenatally and lactationally exposed to a reconstituted PCB mixture. Environ. Toxicol. 29, 856-866. doi: 10.1002/tox.21812

Buchanan, G., Yang, M., Cheong, A., Harris, J. M., Irvine, R. A., Lambert, P. F., et al. (2004). Structural and functional consequences of glutamine tract variation in the androgen receptor. Hum. Mol. Genet. 13, 1677-1692. doi: $10.1093 / \mathrm{hmg} / \mathrm{ddh} 181$

Buck-Koehntop, B. A. and Defossez, P. A., (2013). On how mammalian transcription factors recognize methylated DNA. Epigenetics 8, 131-137. doi: 10.4161/epi.23632

Carthew, R. W., and Sontheimer, E. J. (2009). Origins and Mechanisms of miRNAs and siRNAs. Cell 136, 642-655. doi: 10.1016/j.cell.2009.01.035

Casati, L. (2013). Epigenetics and PCBs: Commentary to "Androgen receptor activation by polychlorinated biphenyls: epigenetic effects mediated by the histone demethylase Jarid1b." Endocrine Disruptors 1, 4. doi: 10.4161/endo. 27347

Casati, L., Colciago, A., and Celotti, F. (2010). Epigenetic mechanisms in health and diseases. Brasília Med. 48, 209-218.

Casati, L., Sendra, R., Colciago, A., Negri-Cesi, P., Berdasco, M., Esteller, M., et al. (2012). Polychlorinated biphenyls affect histone modification pattern in early development of rats: a role for androgen receptor-dependent modulation? Epigenomics 4, 101-112. doi: 10.2217/epi.11.110

Casati, L., Sendra, R., Poletti, A., Negri-Cesi, P., and Celotti, F. (2013). Androgen receptor activation by polychlorinated biphenyls: epigenetic effects mediated by the histone demethylase Jarid1b. Epigenetics 8, 1061-1068. doi: 10.4161/epi.25811

Cedar, H., and Bergman, Y. (2009). Linking DNA methylation and histone modification: patterns and paradigms. Nat. Rev. Genet. 10, 295-304. doi: $10.1038 / \operatorname{nrg} 2540$

Cheung, P., and Lau, P. (2005). Epigenetic regulation by histone methylation and histone variants. Mol. Endocrinol. 19, 563-573. doi: 10.1210/me.2004-0496

Chi, P., Allis, C. D., and Wang, G. G. (2010). Covalent histone modificationsmiswritten, misinterpreted and mis-erased in human cancers. Nat. Rev. Cancer 10, 457-469. doi: $10.1038 / \mathrm{nrc} 2876$

Clouaire, T., and Stancheva, I. (2008). Methyl-CpG binding proteins: specialized transcriptional repressors or structural components of chromatin? Cell. Mol. Life Sci. 65, 1509-1522. doi: 10.1007/s00018-008-7324-y

Colciago, A., Casati, L., Mornati, O., Vergoni, A. V., Santagostino, A., Celotti, F., et al. (2009). Chronic treatment with polychlorinated biphenyls (PCB) during pregnancy and lactation in the rat Part 2: Effects on reproductive parameters, on sex behavior, on memory retention and on hypothalamic expression of aromatase and 5alpha-reductases in the offspring. Toxicol. Appl. Pharmacol. 239, 46-54. doi: 10.1016/j.taap.2009.04.023

Colciago, A., Negri-Cesi, P., Pravettoni, A., Mornati, O., Casati, L., and Celotti, F. (2006). Prenatal Aroclor 1254 exposure and brain sexual differentiation: effect on the expression of testosterone metabolizing enzymes and androgen receptors in the hypothalamus of male and female rats. Reprod. Toxicol. 22, 738-745. doi: 10.1016/j.reprotox.2006.07.002

Crews, D., Gillette, R., Scarpino, S. V., Manikkam, M., Savenkova, M. I., and Skinner, M. K. (2012). Epigenetic transgenerational inheritance of altered stress responses. Proc. Natl. Acad. Sci. U.S.A. 109, 9143-9148. doi: 10.1073/pnas.1118514109

Desaulniers, D., Xiao, G. H., Lian, H., Feng, Y. L., Zhu, J., Nakai, J., et al. (2009). Effects of mixtures of polychlorinated biphenyls, methylmercury, and organochlorine pesticides on hepatic DNA methylation in prepubertal female Sprague-Dawley rats. Int. J. Toxicol. 28, 294-307. doi: $10.1177 / 1091581809337918$

Dolinoy, D. C., Weidman, J. R., Waterland, R. A., and Jirtle, R. L. (2006). Maternal genistein alters coat color and protects Avy mouse offspring from obesity by modifying the fetal epigenome. Environ. Health Perspect. 114, 567-572. doi: 10.1289/ehp. 8700

El Hajj, N., Schneider, E., Lehnen, H., and Haaf, T. (2014). Epigenetics and life-long consequences of an adverse nutritional and diabetic intrauterine environment. Reproduction 148, R111-R120. doi: 10.1530/REP-14-0334

Fang, M., Chen, D., and Yang, C. S. (2007). Dietary polyphenols may affect DNA methylation. J. Nutr. 137, 223S-228S.

Felsenfeld, G., and Groudine, M. (2003). Controlling the double helix. Nature 421, 448-453. doi: 10.1038/nature01411

Fischle, W., Wang, Y., Jacobs, S. A., Kim, Y., Allis, C. D., and Khorasanizadeh, S. (2003). Molecular basis for the discrimination of repressive methyl-lysine marks in histone H3 by Polycomb and HP1 chromodomains. Genes Dev. 17, 1870-1881. doi: 10.1101/gad.1110503

Fleisch, A. F., Wright, R. O., and Baccarelli, A. A. (2012). Environmental epigenetics: a role in endocrine disease? J. Mol. Endocrinol. 49, R61-R67. doi: 10.1530/JME-12-0066

Foley, D. L., Craig, J. M., Morley, R., Olsson, C. A., Dwyer, T., Smith, K., et al. (2009). Prospects for epigenetic epidemiology. Am. J. Epidemiol. 169, 389-400. doi: 10.1093/aje/kwn380

Gao, L., and Alumkal, J. (2010). Epigenetic regulation of androgen receptor signaling in prostate cancer. Epigenetics 5, 100-104. doi: 10.4161/epi.5.2.10778

Gerhauser, C. (2013). Cancer chemoprevention and nutriepigenetics: state of the art and future challenges. Top. Curr. Chem. 329, 73-132. doi: 10.1007/128_2012_360

Guerrero-Bosagna, C., Covert, T. R., Haque, M. M., Settles, M., Nilsson, E. E., Anway, M. D., et al. (2012). Epigenetic transgenerational inheritance of vinclozolin induced mouse adult onset disease and associated sperm epigenome biomarkers. Reprod. Toxicol. 34, 694-707. doi: 10.1016/j.reprotox.2012.09.005

Guerrero-Bosagna, C., Savenkova, M., Haque, M. M., Nilsson, E., and Skinner, M. K. (2013). Environmentally induced epigenetic transgenerational inheritance of altered Sertoli cell transcriptome and epigenome: molecular etiology of male infertility. PLoS ONE 8:e59922. doi: 10.1371/journal.pone.0059922

Heijmans, B. T., Tobi, E. W., Stein, A. D., Putter, H., Blauw, G. J., Susser, E. S., et al. (2008). Persistent epigenetic differences associated with prenatal exposure to famine in humans. Proc. Natl. Acad. Sci. U.S.A. 105, 17046-17049. doi: 10.1073/pnas.0806560105

Hou, L., Zhang, X., Wang, D., and Baccarelli, A. (2012). Environmental chemical exposures and human epigenetics. Int. J. Epidemiol. 41, 79-105. doi: 10.1093/ije/dyr 154

Jennings, B. A., and Willis, G. (2015). How folate metabolism affects colorectal cancer development and treatment; a story of heterogeneity and pleiotropy. Cancer Lett. 356, 224-230. doi: 10.1016/j.canlet.2014.02.024

Jensen, T. J., Novak, P., Eblin, K. E., Gandolfi, A. J., and Futscher, B. W. (2008). Epigenetic remodeling during arsenical-induced malignant transformation. Carcinogenesis 29, 1500-1508. doi: 10.1093/carcin/bgn102

Kala, R., Peek, G. W., Hardy, T. M., and Tollefsbol, T. O. (2013). MicroRNAs: an emerging science in cancer epigenetics. J. Clin. Bioinforma. 3:6. doi: 10.1186/2043-9113-3-6

Kanherkar, R. R., Bhatia-Dey, N., and Csoka, A. B. (2014). Epigenetics across the human lifespan. Front. Cell Dev. Biol. 2:49. doi: 10.3389/fcell.2014.00049

Kollara, A., and Brown, T. J. (2010). Four and a half LIM domain 2 alters the impact of aryl hydrocarbon receptor on androgen receptor transcriptional activity. J. Steroid Biochem. Mol. Biol. 118, 51-58. doi: 10.1016/j.jsbmb.2009.09.017

Kulis, M., and Esteller, M. (2010). DNA methylation and cancer. Adv. Genet. 70, 27-56. doi: 10.1016/b978-0-12-380866-0.60002-2

Lambrot, R., Xu, C., Saint-Phar, S., Chountalos, G., Cohen, T., Paquet, M., et al. (2013). Low paternal dietary folate alters the mouse sperm epigenome and 
is associated with negative pregnancy outcomes. Nat. Commun. 4, 2889. doi: $10.1038 /$ ncomms 3889

Leader, J. E., Wang, C., Fu, M., and Pestell, R. G. (2006). Epigenetic regulation of nuclear steroid receptors. Biochem. Pharmacol. 72, 1589-1596. doi: 10.1016/j.bcp.2006.05.024

Lee, Y. W., Klein, C. B., Kargacin, B., Salnikow, K., Kitahara, J., Dowjat, K., et al. (1995). Carcinogenic nickel silences gene expression by chromatin condensation and DNA methylation: a new model for epigenetic carcinogens. Mol. Cell. Biol. 15, 2547-2557.

Leonard, S. S., Bower, J. J., and Shi, X. (2004). Metal-induced toxicity, carcinogenesis, mechanisms and cellular responses. Mol. Cell. Biochem. 255, 3-10. doi: 10.1023/B:MCBI.0000007255.72746.a6

Leon-Olea, M., Martyniuk, C. J., Orlando, E. F., Ottinger, M. A., Rosenfeld, C. S., Wolstenholme, J. T., et al. (2014). Current concepts in neuroendocrine disruption. Gen. Comp. Endocrinol. 203, 158-173. doi: 10.1016/j.ygcen.2014.02.005

Lewis, B. P., Burge, C. B., and Bartel, D. P. (2005). Conserved seed pairing, often flanked by adenosines, indicates that thousands of human genes are microRNA targets. Cell 120, 15-20. doi: 10.1016/j.cell.2004.12.035

Lister, R., Pelizzola, M., Dowen, R. H., Hawkins, R. D., Hon, G., Tonti-Filippini, J., et al. (2009). Human DNA methylomes at base resolution show widespread epigenomic differences. Nature 462, 315-322. doi: 10.1038/nature08514

Luger, K., Mader, A. W., Richmond, R. K., Sargent, D. F., and Richmond, T. J. (1997). Crystal structure of the nucleosome core particle at 2.8 A resolution. Nature 389, 251-260. doi: 10.1038/38444

Manikkam, M., Haque, M. M., Guerrero-Bosagna, C., Nilsson, E. E., and Skinner, M. K. (2014). Pesticide methoxychlor promotes the epigenetic transgenerational inheritance of adult-onset disease through the female germline. PLoS ONE 9:e102091. doi: 10.1371/journal.pone.0102091

Marsit, C. J., Eddy, K., and Kelsey, K. T. (2006). MicroRNA responses to cellular stress. Cancer Res. 66, 10843-10848. doi: 10.1158/0008-5472.CAN-06-1894

McGowan, P. O., Sasaki, A., D’Alessio, A. C., Dymov, S., Labonte, B., Szyf, M., et al. (2009). Epigenetic regulation of the glucocorticoid receptor in human brain associates with childhood abuse. Nat. Neurosci. 12, 342-348. doi: $10.1038 / \mathrm{nn} .2270$

McGowan, P. O., Sasaki, A., Huang, T. C., Unterberger, A., Suderman, M., Ernst, C., et al. (2008). Promoter-wide hypermethylation of the ribosomal RNA gene promoter in the suicide brain. PLoS ONE 3:e2085. doi: 10.1371/journal.pone.0002085

Newbold, R. R. (2011). Developmental exposure to endocrine-disrupting chemicals programs for reproductive tract alterations and obesity later in life. Am. J. Clin. Nutr. 94, 1939S-1942S. doi: 10.3945/ajcn.110. 001057

Patel, D. J., and Wang, Z. (2013). Readout of epigenetic modifications. Annu. Rev. Biochem. 82, 81-118. doi: 10.1146/annurev-biochem-072711-165700

Portigal, C. L., Cowell, S. P., Fedoruk, M. N., Butler, C. M., Rennie, P. S., and Nelson, C. C. (2002). Polychlorinated biphenyls interfere with androgeninduced transcriptional activation and hormone binding. Toxicol. Appl. Pharmacol. 179, 185-194. doi: 10.1006/taap.2002.9371

Razin, A. (1998). CpG methylation, chromatin structure and gene silencing-a three-way connection. EMBO J. 17, 4905-4908. doi: 10.1093/emboj/17.17.4905

Reichard, J. F., Schnekenburger, M., and Puga, A. (2007). Long term low-dose arsenic exposure induces loss of DNA methylation. Biochem. Biophys. Res. Commun. 352, 188-192. doi: 10.1016/j.bbrc.2006.11.001

Reik, W., Dean, W., and Walter, J. (2001). Epigenetic reprogramming in mammalian development. Science 293, 1089-1093. doi: $10.1126 /$ science. 1063443

Rivera, C. M., and Ren, B. (2013). Mapping human epigenomes. Cell 155, 39-55. doi: 10.1016/j.cell.2013.09.011

Sato, K., Fukata, H., Kogo, Y., Ohgane, J., Shiota, K., and Mori, C. (2009). Neonatal exposure to diethylstilbestrol alters expression of DNA methyltransferases and methylation of genomic DNA in the mouse uterus. Endocr. J. 56, 131-139. doi: 10.1507/endocr..K08E-239

Schulz, L. C. (2010). The Dutch Hunger Winter and the developmental origins of health and disease. Proc. Natl. Acad. Sci. U.S.A. 107, 16757-16758. doi: 10.1073/pnas. 1012911107

Singh, S., and Li, S. S. (2012). Epigenetic effects of environmental chemicals bisphenol a and phthalates. Int. J. Mol. Sci. 13, 10143-10153. doi: 10.3390/ijms130810143
Skinner, M. K., Guerrero-Bosagna, C., Haque, M., Nilsson, E., Bhandari, R., and McCarrey, J. R. (2013). Environmentally induced transgenerational epigenetic reprogramming of primordial germ cells and the subsequent germ line. PLoS ONE 8:e66318. doi: 10.1371/journal.pone.0066318

Skinner, M. K., Rawls, A., Wilson-Rawls, J., and Roalson, E. H. (2010). Basic helixloop-helix transcription factor gene family phylogenetics and nomenclature. Differentiation 80, 1-8. doi: 10.1016/j.diff.2010.02.003

Stouder, C., and Paoloni-Giacobino, A. (2010). Transgenerational effects of the endocrine disruptor vinclozolin on the methylation pattern of imprinted genes in the mouse sperm. Reproduction 139, 373-379. doi: 10.1530/REP-09-0340

Suganuma, T. and Workman, J. L. (2011). Signals and combinatorial functionsof histone modifications. Annu. Rev. Biochem. 80, 473-499. doi: 10.1146/annurevbiochem-061809-175347

Suzuki, E., Zhao, Y., Ito, S., Sawatsubashi, S., Murata, T., Furutani, T., et al. (2009). Aberrant E2F activation by polyglutamine expansion of androgen receptor in SBMA neurotoxicity. Proc. Natl. Acad. Sci. U.S.A. 106, 3818-3822. doi: 10.1073/pnas.0809819106

Szyf, M. (2009). The early life environment and the epigenome. Biochim. Biophys. Acta 1790, 878-885. doi: 10.1016/j.bbagen.2009.01.009

Tabb, M. M., and Blumberg, B. (2006). New modes of action for endocrinedisrupting chemicals. Mol. Endocrinol. 20, 475-482. doi: 10.1210/me.2004-0513

Tobi, E. W., Lumey, L. H., Talens, R. P., Kremer, D., Putter, H., Stein, A. D., et al. (2009). DNA methylation differences after exposure to prenatal famine are common and timing- and sex-specific. Hum. Mol. Genet. 18, 4046-4053. doi: $10.1093 / \mathrm{hmg} / \mathrm{ddp} 353$

Tollefsbol, T. O. (2014). Dietary epigenetics in cancer and aging. Cancer Treat. Res. 159, 257-267. doi: 10.1007/978-3-642-38007-5_15

Vismara, G., Simonini, F., Onesto, E., Bignamini, M., Miceli, V., Martini, L., et al. (2009). Androgens inhibit androgen receptor promoter activation in motor neurons. Neurobiol. Dis. 33, 395-404. doi: 10.1016/j.nbd.2008.11.007

Weaver, I. C., Diorio, J., Seckl, J. R., Szyf, M., and Meaney, M. J. (2004). Early environmental regulation of hippocampal glucocorticoid receptor gene expression: characterization of intracellular mediators and potential genomic target sites. Ann. N.Y. Acad. Sci. 1024, 182-212. doi: 10.1196/annals.1321.099

Wilson, V. S., Blystone, C. R., Hotchkiss, A. K., Rider, C. V., and Gray, L. E. Jr. (2008). Diverse mechanisms of anti-androgen action: impact on male rat reproductive tract development. Int. J. Androl. 31, 178-187. doi: 10.1111/j.1365-2605.2007.00861.x

Wong, R. L., and Walker, C. L. (2013). Molecular pathways: environmental estrogens activate nongenomic signaling to developmentally reprogram the epigenome. Clin. Cancer Res. 19, 3732-3737. doi: 10.1158/1078-0432.CCR-130021

Xiang, Y., Zhu, Z., Han, G., Ye, X., Xu, B., Peng, Z., et al. (2007). JARID1B is a histone $\mathrm{H} 3$ lysine 4 demethylase up-regulated in prostate cancer. Proc. Natl. Acad. Sci. U.S.A. 104, 19226-19231. doi: 10.1073/pnas.0700735104

Zhang, H., and Zhu, J. K. (2012). Active DNA demethylation in plants and animals. Cold Spring Harb. Symp. Quant. Biol. 77, 161-173. doi: 10.1101/sqb.2012.77.014936

Zhang, X., and Ho, S. M. (2011). Epigenetics meets endocrinology. J. Mol. Endocrinol. 46, R11-R32. doi: 10.1677/JME-10-0053

Zhang, Y., and Chen, H. (2011). Genistein, an epigenome modifier during cancer prevention. Epigenetics 6, 888-891. doi: 10.4161/epi.6.7.16315

Zoroddu, M. A., Peana, M., Medici, S., Casella, L., Monzani, E., and Costa, M. (2010). Nickel binding to histone H4. Dalton Trans. 39, 787-793. doi: 10.1039/B916019C

Conflict of Interest Statement: The Reviewer Dr. Adriana Maggi declares that, despite sharing an affiliation with the authors, Dr. Lavinia Casati and Dr. Fabio Celotti, the review process was handled objectively and no conflict of interest exists. The authors declare that the research was conducted in the absence of any commercial or financial relationships that could be construed as a potential conflict of interest.

Copyright (๑) 2015 Casati, Sendra, Sibilia and Celotti. This is an open-access article distributed under the terms of the Creative Commons Attribution License (CC BY). The use, distribution or reproduction in other forums is permitted, provided the original author(s) or licensor are credited and that the original publication in this journal is cited, in accordance with accepted academic practice. No use, distribution or reproduction is permitted which does not comply with these terms. 